\section{Es tiempo de centrarse en los cuidados no remunerados}

\section{It is time to focus on unpaid caregiving}

\section{Sr. Editor:}

En todo el mundo, a niñas, jóvenes y mujeres se les asigna la provisión de cuidados no remunerados en sus familias y comunidades en forma arbitraria y diferencial, naturalizando la atención no remunerada de personas dependientes o con algún grado de discapacidad $^{1}$. De tal modo que, tareas como la asistencia con las actividades de la vida diaria, cuidados materiales, económicos o psicológicos, y el sostén del vínculo con el sistema de salud, para la realización del efectivo derecho a la salud de las personas cuidadas ${ }^{1}$, han pasado a ser elementos constitutivos de la experiencia del ser mujer.

Lo anterior explicita el vínculo entre el género, la salud y el desarrollo sostenible, el que ha sido reconocido por las Naciones Unidas, y explicitado en compromisos internacionales como la Cuarta Conferencia Mundial sobre la Mujer en Beijing ${ }^{2}$, que en la década 1990-99 llamó a la incorporación urgente del trabajo no remunerado de las mujeres en las estadísticas económicas y vitales, considerándolo capital para el sostenimiento de la vida y en la lucha contra la pobreza, emplazando a los países miembros a promover la igualdad de género.

El año 2008, la Oficina Regional para las Américas de la Organización Mundial de la Salud (OPS/OMS), en concordancia con la integración de una perspectiva de género en la labor de la OMS, publicó una "Guía para el análisis y el monitoreo de la equidad de género en las políticas de salud"3, marco de referencia para evaluar el grado en que las políticas de salud de los países de la región integran transversalmente un enfoque de equidad de género. Este documento destaca entre sus áreas de observación la medición del trabajo no remunerado en salud, los riesgos ocupacionales para la salud que se le asocian y las acciones que se han previsto para enfrentar tales riesgos ${ }^{3}$.

En concordancia con la relevancia que organismos internacionales han otorgado al trabajo no remunerado, distintos países han desarrollado métodos para contabilizar el valor financiero de los cuidados no remunerados. En el año 2011, la Asociación Americana de Personas Retiradas (AARP), de los Estados Unidos de Norteamérica, publicó un informe en el que señaló que la contribución económica de cuidadores familiares no remunerados, que atendían a adultos con limitaciones en actividades de la vida diaria se podía estimar, para el año 2009, en US $\$ 4.500$ millones, equivalente a 3,2\% del Producto Interno Bruto (PIB) de los Estados Unidos".

El Análisis Global para la Valorización de lo Invaluable ${ }^{1}$ corresponde a un estudio reciente y único, ya que aplicó los métodos de costos de oportunidad y de bienes sustitutivos en una base de datos de 32 países, contabilizando a 52\% de la población mundial, para determinar la contribución de las mujeres al sector salud. Tomando en cuenta las diferencias salariales por género y los beneficios de la seguridad social, el trabajo total vinculado al sector salud realizado por las mujeres se estimó en 7,04\% del PIB mundial del año 2010, los cuidados no remunerados representaban $43,8 \%$ de ese total ${ }^{1}$.

En Chile, las cuentas nacionales omiten el trabajo no remunerado, aun cuando el Estado ha suscrito y ratificado compromisos relativos al empoderamiento de la mujer. Los datos internacionales hacen suponer que la magnitud de la contribución de las mujeres al sector salud permitiría hablar de un "segundo" sistema de salud que opera de forma invisible, siendo congruente con otros indicadores del país que destacan la feminización de los cuidados no remunerados, como, por ejemplo, el que las obligaciones familiares (cuidado de otros familiares) sean referidas como la principal causa de inactividad laboral entre las mujeres ${ }^{5}$.

No está de más mencionar que la falta de reconocimiento de los cuidados no remunerados puede traer costos de oportunidad incalculables para las mujeres, sus familias, y la sociedad en general, facilitando la transmisión intergeneracional de la pobreza, interrumpiendo trayectorias educativas y laborales desde temprana edad. $\mathrm{Al}$ respecto, en el Reino Unido, un estudio longitudinal de dos décadas ha determinado con precisión que el género y las actitudes sociales son instrumentales en moldear las trayectorias vitales ligadas al trabajo remunerado y no remunerado, y que los cuidadores no remunerados suelen terminar más pobres, menos felices y menos saludables que su contraparte ${ }^{6}$.

Es tiempo de visibilizar los cuidados no remunerados, contabilizarlos y evaluar los costos que tienen para quienes en su mayoría son las encargadas de efectuarlos, las mujeres. Es tiempo de interrogarse por el grado de integración alcanzado entre ambos sistemas de salud, el formal, institucionalizado, y el informal, a cargo de mujeres. Las ciencias de la salud en Chile deben desempeñar un rol crucial en la necesaria visibilidad de esta realidad, sobre todo considerando la relevancia que se le ha otorgado a modelos de salud centrados en la comunidad que, para afrontar los desafíos propios de la transición epidemiológica, descansan en gran medida sobre la participación y responsabilidad familiar en salud.

Pablo Martínez ${ }^{1,2,3}$, Irene Magaña ${ }^{4}$, M. Soledad Loyola ${ }^{5}$ ${ }^{1}$ Departamento de Psiquiatría y Salud Mental, Hospital Clínico Universidad de Chile, Santiago, Chile. ${ }^{2}$ Instituto Milenio para la Investigación en Depresión y Personalidad, Santiago, Chile. ${ }^{3}$ Centro de Innovación en Tecnologías de la Información para Aplicaciones Sociales CITIAPS, Universidad de Santiago de Chile, Santiago, Chile.

${ }^{4}$ Escuela de Psicología, Facultad de Humanidades, 
Universidad de Santiago de Chile. ${ }^{5}$ Laboratorio de Investigación Virtual Lab-Usach, Universidad de Santiago de Chile, Santiago, Chile.

\section{Referencias}

1. Langer A, Meleis A, Knaul FM, Atun R, Aran M, Binagwaho A, et al. The Lancet Commissions Women and Health: the key for sustainable development. Lancet 2015; 6736 (15): 1-45.

2. Naciones Unidas. Informe de la Cuarta Conferencia Mundial sobre la Mujer. Beijing, 4 a 15 de septiembre de 1995. Nueva York: Naciones Unidas; 1996. 232 p.

3. Organización Panamericana de la Salud. Guía para el análisis y el monitoreo de la equidad de género en las políticas de salud. Washington, D.C.: Organización Panamericana de la Salud; 2008. 95 p.

4. Feinberg L, Reinhard SC, Houser A, Choula R. Valuing the Invaluable: 2011 Update. The Growing Contributions and Costs of Family Caregiving. American Association of Retired Persons [AARP] Public Policy Institute; 2011. 28 p.
5. Instituto Nacional de Estadísticas, Departamento de Estudios Laborales y Departamento de Estudios Sociales (Chile). Mujeres en Chile y el mercado del trabajo: participación laboral femenina y brechas salariales. Santiago de Chile: Instituto Nacional de Estadísticas (Chile); 2015.

6. Carmihael F, Ercolani MG. Unpaid caregiving and paid work over life-courses: different pathways, diverging outcomes. Social Science \& Medicine 2016; 156: 1-11.

Fuente de apoyo financiero: proyecto DICYT 031693MF de la Vicerrectoría de Investigación e Innovación de la Universidad de Santiago de Chile. Esta fuente de financiamiento no tuvo ningún rol en el diseño del estudio, o en la recolección, análisis e interpretación de los datos, ni tampoco en la redacción y envío del manuscrito. Este estudio recibió el apoyo del Fondo de Innovación para la Competitividad (IC) del Ministerio de Economía, Fomento y Turismo, a través de la Iniciativa Científica Milenio. Proyecto IS 130005.

Correspondencia a:

Pablo Martínez.

Avenida La Paz 1003, Recoleta, Santiago, Chile. +56 227182385. pablo.martinezd@usach.cl 